\title{
Liquid PTVA: a faster and cheaper alternative for generating multi-copy clones in Pichia pastoris
}

Rochelle $A w^{1,2^{*}}$ and Karen M. Polizzi ${ }^{1,2^{*}}$

\begin{abstract}
Background: Multiple cognate gene copy clones have often been used in order to increase the yield of recombinant protein expression in the yeast Pichia pastoris. The method of posttransformational vector amplification (PTVA) has allowed for the efficient generation of multi-copy clones in P. pastoris. However, despite its relative ease and success, this process can be expensive and time consuming.

Results: We have developed a modified version of PTVA, called Liquid PTVA, which allows for faster and cheaper selection of multi-copy clones. Cultures are grown in liquid medium with only a final selection carried out on agar plates, reducing overall antibiotic usage and increasing the speed of clone amplification. In addition, it was established that starting PTVA with a single copy clone resulted in higher copy number strains for both traditional plate PTVA and liquid PTVA. Furthermore, using the Zeocin selection marker in liquid PTVA results in strains with higher growth rates, which could be beneficial for recombinant protein production processes.
\end{abstract}

Conclusions: We present a methodology for creating multi-copy clones that can be achieved over 12 days instead of the traditional 45 and at approximately half the cost.

Keywords: Pichia pastoris/Komagataella phaffii, Multi-copy clones, Posttransformational vector amplification, Recombinant protein expression

\section{Background}

Pichia pastoris has been used for over 30 years to produce recombinant proteins with expression levels of select proteins reaching up to $20 \mathrm{~g} \mathrm{~L}^{-1}[1,2]$. P. pastoris is an ideal industrial cell factory due to its ability to reach very high cell densities and secrete proteins into the supernatant, which coupled with a low level of native proteins, eases downstream processing [3]. A popular way to create strains with increased protein production is to increase the number of cognate genes $[4,5]$. This is particularly effective with intracellular expression of proteins $[6,7]$, but is also a common strategy with secreted proteins $[8,9]$.

\footnotetext{
*Correspondence: r.aw08@imperial.ac.uk; k.polizzi@imperial.ac.uk

${ }^{1}$ Department of Life Sciences, Imperial College London,

London SW7 2AZ, UK

Full list of author information is available at the end of the article
}

While an increase in titer from multi-copy clones has often been reported, there are instances, especially with secreted proteins, where the relationship is not linear (i.e. the highest copy strains do not always give the highest expression) $[5,10]$. This is often attributed to bottlenecks in the secretory pathway [11]. However, the copy number at which secretion saturation occurs is often protein-specific, and as a result strains with different copy numbers must be evaluated in order to identify those with the maximum expression [7]. Furthermore, increasingly strain engineering efforts have aimed to expand the capacity of the secretory pathway, e.g. by overexpressing accessory proteins to aid in protein folding $[12,13]$. Such research relies on testing expression at a range of copy numbers to show the effect of the engineering efforts on the titer obtained. Thus, there is a need for a rapid and reliable method to generate strains with a range of gene copy number. 
There are several established experimental methods for generating multi-copy clones including in vitro multimerization of the vector before transformation and direct selection of transformants on high concentrations of antibiotics, made possible by the increased use of Zeocin and the modification of the Tn903kanr gene meaning pre-selection using histidine auxotrophy is not required [14]. With the direct selection method, the number of colonies generated on plates containing higher concentration of antibiotics is often severely reduced, limiting the number of multi-copy strains obtained. However, a higher proportion of the surviving population will be multi-copy clones and so such experiments can still be used to generate strains with a range of copy number.

Due to the low efficiency of generating multi-copy clones via direct selection, in 2008 Sunga et al. proposed the method of posttransformational vector amplification (PTVA). In PTVA, instead of direct selection on high concentrations of antibiotic, cells are spotted onto agar plates with increasing antibiotic concentrations with approximately 5 days growth in between each step [15]. During the outgrowth phase, the copy number of the antibiotic resistance gene is increased to allow the cell to adapt to the higher antibiotic concentration. Using Southern blot, it was demonstrated that cells actually amplify the entire cassette, including the gene of interest. Thus, strains that survive at higher antibiotic concentrations also contain a higher number of intact copies of the gene of interest. The advantage of using PTVA over direct selection is that the frequency of "jackpot" clones, those with over 10 copies, increases from $1-2$ to $5-6 \%$ [15].

PTVA has been widely adopted by the P. pastoris community with many studies using it for comparison of titers from strains of different copy number [7, 16, 17]. However, despite the apparent ease of PTVA, the methodology can be time consuming and laborious, not to mention expensive, particularly when Zeocin is used as a selection agent. Herein, we describe a method to reduce the time and cost to carry out PTVA through serial passaging in liquid medium, which still results in a wide range of strains containing different copy numbers.

\section{Results and Discussion}

Liquid PTVA results in multi-copy clones with saturated GFP expression

Liquid PTVA with a medium change every 12 h versus Plate PTVA

Initially four individual vectors were designed: pZGFP, pZ $\alpha$ GFP, pKGFP and $\mathrm{pK} \alpha \mathrm{GFP}$, all expressing the green fluorescent protein (GFP) under the control of the alcohol oxidase 1 (AOX1) promoter (Additional file 1: Figure S1). pZGFP and pZ $\alpha$ GFP utilize the commercial pPICZ and pPICZ $\alpha$ vectors from Invitrogen, respectively, while pKGFP and pK $\alpha$ GFP use the pKANB and pKAN $\alpha B$ vectors, respectively [14]. Two different vector backbones were used to test whether this method works with both Zeocin and G418 selection, as was demonstrated in the original paper [15]. Furthermore, multi-copy clones have been shown to linearly increase the titer of intracellular, but not secreted, proteins [5]. Therefore it was interesting to compare the effect of producing GFP as an intracellular protein (pZGFP and pKGFP) or as a secreted protein (pZ $\alpha$ GFP and $\mathrm{pK} \alpha \mathrm{GFP})$ using the $\alpha$-mating factor $(\alpha-\mathrm{MF})$ from Saccharomyces cerevisiae to direct the protein to the secretory pathway.

All four vectors were transformed into $P$. pastoris and single colonies were selected, expressed in microtiter plates and run on a SDS-PAGE gel to ensure that GFP expression was occurring (data not shown). The $\Delta k u 70$ strain was selected to reduce non-homologous recombination and target the gene of interest to the desired locus (in this case the $A O X 1$ locus), preventing off target integration of non-functional copies at other sites, which might skew the analysis [18]. Since it has been hypothesized that PTVA utilizes homologous recombination mechanisms, this knockout should have no impact on the PTVA process itself.

The PTVA procedures are outlined in Fig. 1. Initially colonies that showed the highest level of expression were selected to undergo both traditional PTVA and liquid PTVA where the medium was changed every $12 \mathrm{~h}$ (L12). Copy number of the initial clones was determined by qPCR, because clones with different starting copy number can be obtained by transformation, even when plating on low concentrations of antibiotic.

For ease of interpretation each strain is denoted with a number to indicate the initial starting copy number. pZGFP was identified as a single copy clone (pZGFP$1), p Z \alpha G F P$ as a three copy clone (pZ $\alpha$ GFP-3), and pKGFP and pK $\alpha$ GFP as two copy clones (pKGFP-2 and $\mathrm{pK} \alpha \mathrm{GFP}-2)$. For liquid PTVA colonies were inoculated into YPD medium containing the initial antibiotic concentration used for transformation $\left(100 \mu \mathrm{g} \mathrm{mL} \mathrm{m}^{-1}\right.$ Zeocin and $\left.350 \mu \mathrm{g} \mathrm{mL}^{-1} \mathrm{G} 418\right)$. Thereafter cultures were centrifuged every $12 \mathrm{~h}$ and the medium replaced with YPD containing the next sequentially higher concentration as indicated in the materials and methods. After the cultures were grown for $12 \mathrm{~h}$ in the presence of the highest concentration of antibiotic $\left(3000 \mu \mathrm{g} \mathrm{mL} \mathrm{m}^{-1}\right.$ for both Zeocin and G418), the cultures were diluted by $10^{5}$ and plated onto YPD agar plates maintaining selection at $3000 \mu \mathrm{g} \mathrm{mL}{ }^{-1}$.Traditional PTVA was carried out as previously described by Sunga et al., including the initial step which requires streaking to single colony [15]; however to ensure colonies were monoclonal before analysis an 


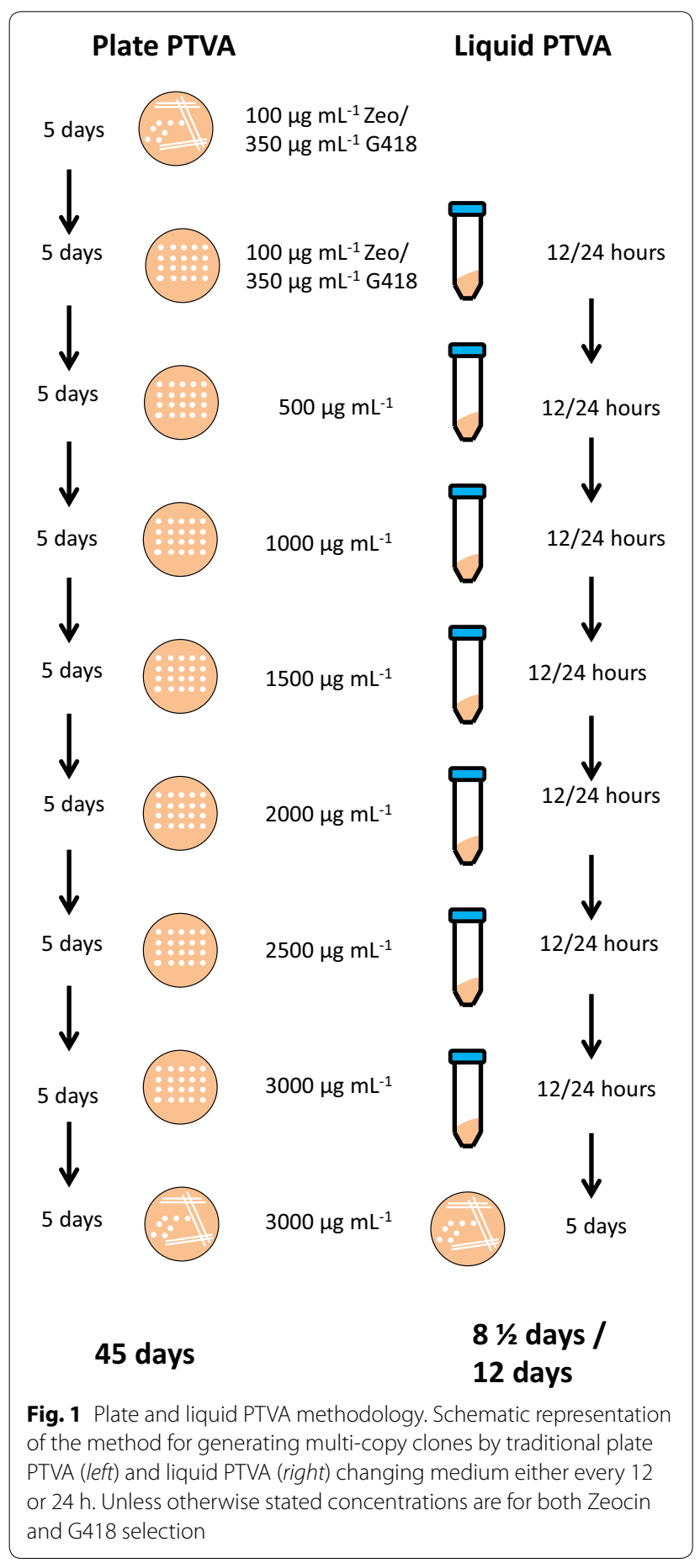

additional step was added where the spot from the final plate was streaked to single colonies.

The first observation was that no colonies were isolated from the pZ $\alpha$ GFP- 3 culture in the final plating step despite the highest rate of growth during the liquid PTVA experiment, in particular at the lower concentrations of antibiotic. Even though significant cell mass had accumulated over the course of the experiment, growth occurred in the initial stages at low antibiotic concentrations and the cells were non-viable after failing to adapt to the higher concentrations of antibiotics. The liquid PTVA procedure was repeated; however again no colonies were observed (data not shown). From the remaining three conditions a similar number of colonies were obtained $\left(\sim 10^{7}\right)$ from which twenty individual colonies were selected for analysis from both the traditional and L12 PTVA final plates. Each colony was analysed by qPCR to determine copy number (Fig. 2).

For pZGFP-1 (Fig. 2a) the median copy number for strains produced by plate PTVA was significantly higher than that for L12 with an average of 10 copies and 6.8 copies, respectively $(\mathrm{p}=0.0123)$. Furthermore, plate PTVA yielded the strain with the highest individual copy number (37) compared to 18 for L12 and had a significantly larger variation in copy number $(\mathrm{p}=0.0119)$. Despite this, both methods produced a large range of clones with varying copy numbers that should be suitable for conducting a study on the impact of copy number on expression.

The median copy number of pKGFP-2 strains (Fig. 2b) decreased to 0.95 and 1.22 copies for plate and liquid PTVA, respectively, from the initial two copy clone used to start the experiment. Furthermore, none of the strains isolated from L12 had an increased copy number. Plate PTVA did give a small number of strains with a copy number higher than the starting clone (as high as 4). However, statistically there was no significant difference in copy number variance between the two conditions $(\mathrm{p}=0.182)$.

For pKoGFP-2 (Fig. 2c) the median copy number for strains produced by plate PTVA was also lower than the initial starting clone at 1.2; however L12 strains showed a higher median copy number of 3.4. The highest copy clones had 4 and 8 copies of pK $\alpha$ GFP-2 for plate and L12 PTVA, respectively. The increase in both median copy number $\left(\mathrm{p}=8.34 \mathrm{E}^{-8}\right)$ and copy number variance $\left(\mathrm{p}=1.69 \mathrm{E}^{-6}\right)$ for L12 versus plate PTVA strains was statistically significant.

\section{Liquid PTVA with a medium change every $24 h$}

Due to the lack of isolated colonies from L12 with pZ $\alpha$ GFP-3 strains and the fact that liquid PTVA did not consistently result in high copy clones (e.g. pKGFP-1, Fig. $2 \mathrm{~b}$ ), the protocol was modified to change the medium every $24 \mathrm{~h}$ (L24) in order to give the cells more time for copy number expansion before forcing adaptation. Furthermore, this is a more manageable experimental set up for a single person as opposed to changing medium every $12 \mathrm{~h}$. 


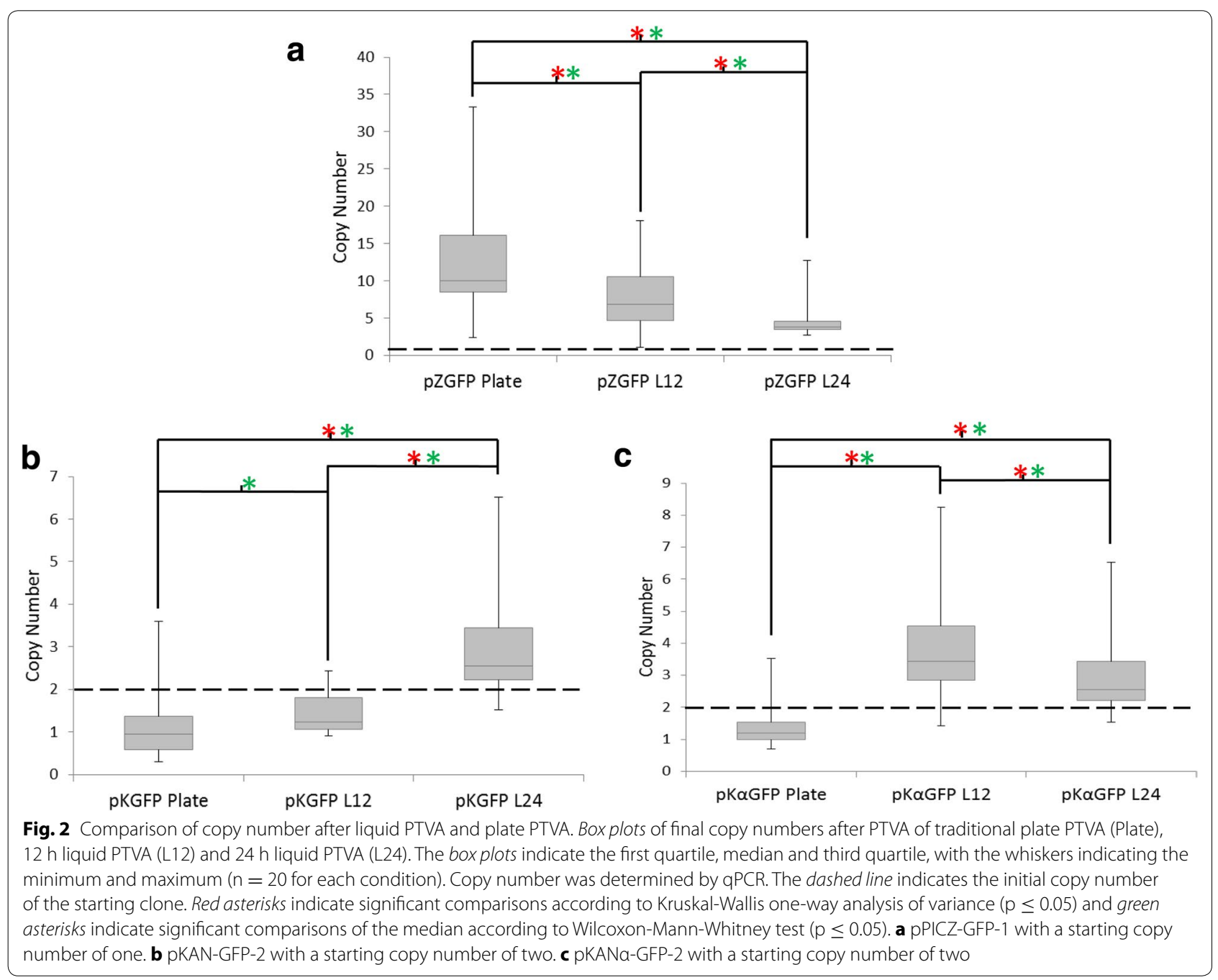

L24 still resulted in no colonies from the pZ $\alpha \mathrm{GFP}-3$ experiment when grown on the final selection plate (data not shown). Again, a similar observation was made that growth occurred quickly in the presence of lower concentrations of Zeocin, but the strains failed to adapt to high concentrations.

Colonies from the pZGFP-1, pKGFP-2 and pKaGFP-2 L24 were selected and analysed by qPCR to determine copy number as described previously (Fig. 2). L24 successfully generated multi-copy clones for all three variants. For pZGFP-1 the median copy number from the L24 experiment is the lowest at 3.7, compared to 6.8 and 9.9 for L12 and plate PTVA, respectively (Fig. 2a) and this difference is statistically significant $\left(\mathrm{p}=0.0402, \mathrm{p}=7.5 \mathrm{E}^{-5}\right.$, respectively). The maximum copy number observed in a L24 strain was 13 copies, which is also lower than the other two methods. For selection with G418, L24 with pKGFP-2 resulted in both higher median copy number and maximum copy number than both plate and L12 PTVA (Fig. 2b, $\mathrm{p}=2.35 \mathrm{E}^{-6}$ and $\mathrm{p}=3.49 \mathrm{E}^{-6}$, respectively). On the other hand, L24 with pK $\alpha \mathrm{GFP}-2$ does not result in clones with higher median copy number than L12 (Fig. 2c).

\section{GFP expression from multi-copy clones generated with all three methods}

Given the variability in copy number obtained with different experimental protocols, we wanted to determine the GFP titer of strains made by different methods since often the primary goal of undertaking PTVA is to achieve higher expression levels. It has been reported that expression can saturate, meaning that sometimes higher copy number strains are not required [5]. Protein expression was determined using a fluorescence plate reader, analysing either the culture supernatant from strains secreting GFP (pZ $\alpha$ GFP and pK $\alpha$ GFP) or the cell pellet for intracellular expression ( $\mathrm{pZGFP}$ and $\mathrm{pKGFP}$ ). The latter were normalized using $\mathrm{OD}_{600}$ values to account for cellular auto-fluorescence. 


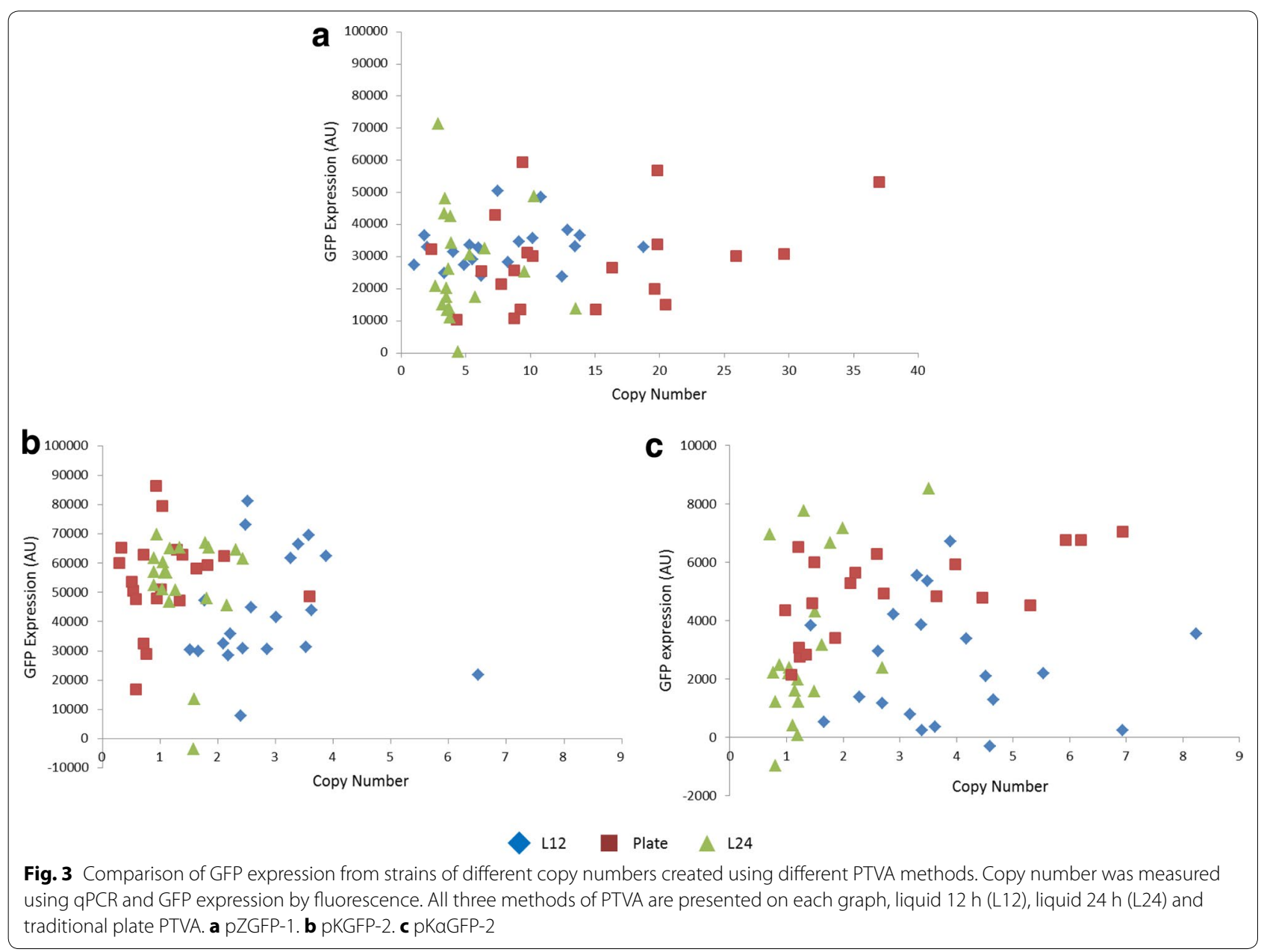

Copy number does not appear to correlate linearly with titer in any of the strains (Fig. 3). For pKaGFP-2, this observation is in line with previous reports that the secretory pathway can saturate such that after a point, increasing copy number no longer results in further increases in titer for extracellular expression $[11,19,20]$. However, it is more surprising that pKGFP-2 and pZGFP1 , do not show a linear correlation between copy number and expression level, because it has been widely reported that for intracellular expression increased copy number correlates with increased titer [5].

However, even though no general trend of increased titer with increased copy number is observed, highly expressing strains can be obtained with any of the PTVA methods. Moreover, using the Kruskal-Wallis one-way analysis of variance it is apparent there is no significant difference $(\mathrm{p}>0.05)$ in the distribution of GFP expression for the strains generated by either L12 or L24 PTVA compared to their plate PTVA counterparts. Therefore, we suggest that it is possible to use any of the PTVA protocols for the generation of multi-copy clones.

\section{Starting with a single copy clone increases the success} of PTVA

In our initial experiments the starting copy number of the strains varied between one and three copies and this appeared to influence the ultimate copy numbers obtained after PTVA. Specifically, the pZGFP-1 strain, which had an initial copy number of one, gave the clone with the highest copy number after PTVA (37), as well as the highest median copy number of all conditions (Fig. 2a). Moreover, the pZaGFP-3 strain, which had an initial copy number of three failed to yield any clones after PTVA at all. In addition, both the pKGFP-2 and pKoGFP-2, which had an initial copy number of two, showed lower copy numbers after PTVA than the pZGFP-1 (Fig. 2b, c). In order to explore the link between starting and final copy numbers further, we used qPCR to select single copy clones of pZ $\alpha$ GFP, pKGFP and pK $\alpha$ GFP and subjected them to traditional plate PTVA and L24.

First, when starting with a single copy clone, we were able to generate colonies with pZaGFP-1 for both plate PTVA and L24 (Fig. 4), in contrast to the 

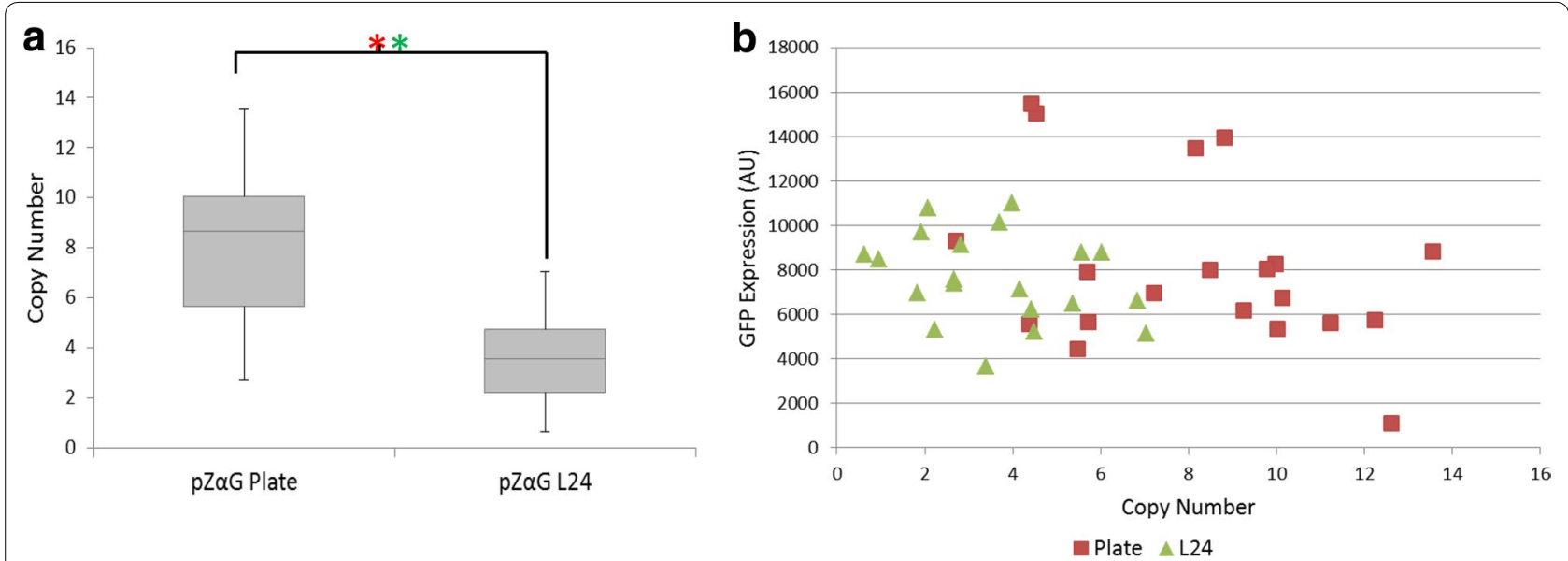

Fig. 4 Liquid PTVA was successful for pZaGFP when starting with a single copy clone. a Box plots of final copy numbers after traditional plate PTVA and liquid PTVA with medium changes every $24 \mathrm{~h}(\mathrm{~L} 24)$ of pZaGFP-1. The box plots indicate the first quartile, median and third quartile, with the whiskers indicating the minimum and maximum ( $n=20$ for each condition). Red asterisks indicate significant comparisons according to KruskalWallis one-way analysis of variance $(p \leq 0.05)$ and green asterisks indicate significant comparisons of the median according to Wilcoxon-Mann-Whitney test ( $p \leq 0.05$ ). Copy number was determined by qPCR. b GFP expression against copy number measured by fluorescence for both traditional plate PTVA and L24

previous experiments. Although median copy number from L24 was not as high as with plate PTVA (3.5 versus 8.6 ), strains with a range of copy numbers were produced with both methods. The highest copy number achieved with L24 was 7 copies, compared to 13 for plate PTVA.

Median and range of GFP expression levels were not significantly different for strains produced by the two methods $(\mathrm{p}=0.881$ and $\mathrm{p}=0.871$ for Wilcoxon-MannWhitney and Kruskal-Wallis $\mathrm{H}$ tests, respectively). It has previously been reported that saturation can occur at varying copy number depending on the protein being expressed. For instance, with trypsinogen saturation occurs at 2 copies and with human serum albumin at $5-7$ copies $[7,10]$. There are no reports of saturation levels with this particular variant of GFP ('superfolder'), but it is apparent from analysing our GFP expression levels (Fig. 3) that a copy number of 7 should be more than sufficient to reach maximum titer (Fig. 4b).

For pKGFP and pK $\alpha$ GFP, it was possible to compare the results of PTVA starting with a two copy strain versus a single copy strain (Fig. 5). Median copy number of strains obtained after PTVA increased when starting with a one copy clone compared to starting with a two copy clone for both PTVA methods. With the pKGFP plasmid, median copy number for strains generated from a one copy clone increased by approximately two copies compared to starting with a two copy clone [2.7 versus 0.9 for plate PTVA and 4.8 versus 2.6 for L24 (Fig. 5a)]. A similar increase was obtained with the pKaGFP plasmid [3.5 versus 1.2 for plate PTVA and 3.9 versus 2.6 for L24
(Fig. 5b)]. Once again there was no correlation between copy number and GFP expression (Additional file 2: Figure S2).

For all three strains tested, it appeared that starting PTVA with a single copy clone resulted in strains with a significantly higher median copy number than when starting with a multi-copy clone. This result was unexpected as the influence of starting copy number has not been previously reported. The observation that single copy clones are better able to expand copy number also supports the theory that our initial pZ $\alpha$ GFP-3 clone was unable to adapt to high concentrations of antibiotic because of a lack of selection pressure in early phases of PTVA.

\section{Liquid PTVA using Zeocin as the selection reagent results in strains with higher growth rates}

Some strains generated in this study showed higher titer at lower copy number than other strains with higher copy number. This was also true for the strains expressing intracellular GFP where secretion saturation should not be an issue. Theoretically, due to the nature of liquid PTVA it was possible that the growth rates of the strains could be affected by the need to overcome rapid exposure to higher concentrations of antibiotic. Therefore, we decided to compare the growth rates of some of the strains generated by plate and L24 PTVA to determine if differing growth rates were responsible for the differences in titer. We selected clones from both G418 and Zeocin experiments to determine whether using different antibiotics affected growth rates. 


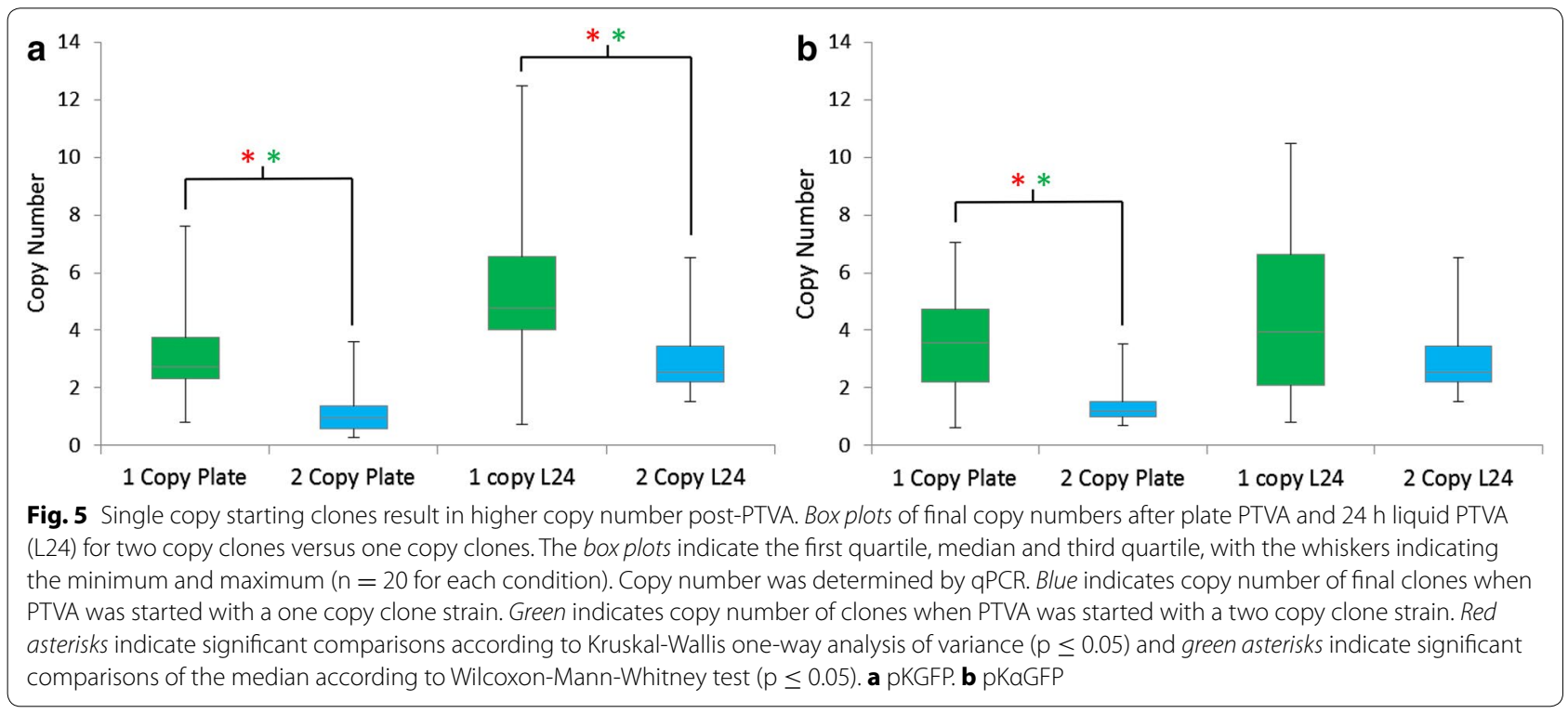

Expression using the $A O X 1$ promoter often begins with growth in glycerol containing medium for $24 \mathrm{~h}$ to accumulate biomass before inducing expression. Hence the cell density after $24 \mathrm{~h}$ will give an indication of the culture density prior to induction using methanol. A higher cell density (a larger number of cells) will increase volumetric productivity.

In order to remove any potential burden effects, clones were selected that were of the same or very similar copy number and titer. Growth rates were assessed by taking optical density measurements $\left(\mathrm{OD}_{600}\right)$ on an hourly basis in a glycerol based medium in the absence of antibiotics, as would be the standard protocol for fed batch production. In addition to the initial $9 \mathrm{~h}$ time course readings, a final $\mathrm{OD}_{600}$ sample was taken after $24 \mathrm{~h}$. Copy number, GFP expression, growth rates and final $\mathrm{OD}_{600}$ readings of the selected clones are shown in Table 1.

From Table 1 the strains produced by a single method do not appear to differ in growth rate. Therefore, neither additional copies of the integrated gene of interest nor additional copies of the resistance gene impact cellular growth. This implies that there is little to no leaky expression from the $A O X 1$ promoter before induction with methanol and that multiple copies of the resistance marker, which is constitutively expressed, do not overburden the cells.

The G418 selection strains show very little variation in growth rates or final $\mathrm{OD}_{600}$. However, the final $\mathrm{OD}_{600}$ of the strains selected via L24 PTVA in Zeocin containing medium are considerably higher than the equivalent plate PTVA strains, as a consequence of a growth rate which is nearly one-third faster. This is likely due to the inherent selection for fast growth in the liquid environment where medium is changed every $24 \mathrm{~h}$, which is absent in plate PTVA where spots are left to grow over a 5 day period. Thus, one advantage of the L24 PTVA method is that it simultaneously selects for strains with faster growth rates and increased copy number. The resulting strains

Table 1 Comparison of growth rates and final $O D_{600}$ readings between paired plate and L24 PTVA strains

\begin{tabular}{|c|c|c|c|c|c|c|c|c|}
\hline \multirow[t]{2}{*}{ Strain } & \multicolumn{4}{|l|}{ Plate PTVA } & \multicolumn{4}{|l|}{ Liquid PTVA } \\
\hline & Copy number & Yield (AU) & Growth rate & Final $O D_{600}$ & Copy number & Yield (AU) & Growth rate & Final $O D_{600}$ \\
\hline pZGFP & 4 & 69,880 & 0.2973 & 59.2 & 4 & 66,829 & 0.4291 & 65.6 \\
\hline pZGFP & 10 & 211,853 & 0.31 & 46 & 10 & 187,676 & 0.4481 & 66.1 \\
\hline pZaGFP & 3 & 9308 & 0.3231 & 46.3 & 3 & 9128 & 0.46 & 57.3 \\
\hline pZaGFP & 7 & 6951 & 0.3228 & 48.2 & 7 & 6609.5 & 0.4283 & 61.7 \\
\hline pKGFP & 2 & 24,068 & 0.3447 & 57.9 & 2 & 27,367 & 0.3474 & 48.4 \\
\hline pKGFP & 6 & 49,650 & 0.3443 & 48.9 & 7 & 51,771 & 0.3426 & 47.7 \\
\hline pKaGFP & 3 & 4643 & 0.3335 & 48.1 & 3 & 4789 & 0.3529 & 50.9 \\
\hline pKaGFP & 7 & 2858 & 0.3491 & 51.1 & 8 & 4000 & 0.3472 & 47.0 \\
\hline
\end{tabular}


will allow a reduction in total protein production time by minimising the initial batch time frames.

One potential explanation for the increased growth rates of strains selected via L24 PTVA with Zeocin, but not those selected via L24 PTVA with G418 is the mechanism of resistance to the antibiotic. Zeocin induces double stranded DNA breaks, whereas G418 blocks polypeptide synthesis by interfering with the $80 \mathrm{~S}$ ribosomal subunit $[21,22]$. Double stranded breaks in the DNA can result in rapid death. However, faster growing cells may be able to adapt more quickly by increasing the amount of resistance protein because of their overall accelerated rate of protein synthesis [23]. In addition, it is possible that double stranded DNA breaks may also facilitate the duplication of the gene cassette during the PTVA process [24].

On the other hand, impairment of ribosomes may have a slower impact on the cell, particularly those that are not actively dividing $[22,25]$. Therefore, slower growing cells are more likely to survive higher concentrations of G418 than faster growing ones. Furthermore it has been reported that in mammalian cells G418 negatively effects growth rate and metabolism [26]. It is also interesting to note that the average copy number of strains selected with G418 is lower than those selected with Zeocin (Fig. 2), which is what might be expected if the selection pressure caused by G418 is weaker. A similar observation has been made in mammalian cells where using a Zeocin resistance cassette resulted in expression of GFP in $100 \%$ of the population, but only $47 \%$ of the population selected with G418 showed expression [27].

\section{Conclusions}

In order to increase the titer of recombinant protein produced in $P$. pastoris, researchers often create strains containing multiple copies of the genes of interest. In addition, for research aimed at increasing the expression capacity of the cell, it is useful to create multi-copy clones with a range of copy numbers in order to systematically evaluate the effects of strain engineering efforts. Therefore a successful, fast and efficient way to do so is required. Various methods have been used historically including plating directly onto selective medium with higher concentrations of antibiotic and in vitro multimerization of the plasmid before transformation [5]. In 2008 Sunga et al. reported a revolutionary method where exposure to a stepwise increase in antibiotic concentration resulted in strains with higher copy number [15]. However, we propose a faster and cheaper alternative to this, which still produces a variety of strains with different copy numbers.

We have shown that it is possible to generate multicopy clones using a liquid PTVA method with medium changes every 12 or $24 \mathrm{~h}$ (L12 or L24, respectively; Figs. 2, 4, 5 and Additional file 3: Table S1). However, L24 resulted in a more robust method that yielded strains with a broader range of copy number for both antibiotics tested (Fig. 2). With L12 multi-copy clones are generated in a total of $8 \frac{1}{2}$ days. The more effective L24 takes a total of 12 days. In contrast, the original method where each plate is left to grow for 5 days, results in a 45 day period to generate multi-copy clones. Even with the longer L24 protocol, the original method takes over three times as long (Fig. 1).

Additionally, because liquid PTVA can be performed in small volumes, the cost of antibiotics is significantly reduced. Overall less than half the amount of antibiotic is required for the liquid PTVA protocol, irrespective of the timing of the medium changes (Table 2).

Surprisingly, in the course of our investigation we discovered that the starting copy number of the initial strain influences the final average copy number and the range of copy numbers generated, regardless of the method of PTVA employed. Starting with a single copy clone results in strains with higher median copy numbers post-PTVA than starting with a multi-copy clone, for both liquid and plate PTVA (Fig. 5). To our knowledge, this observation has not previously been reported. Therefore, when beginning PTVA initial colonies should not be selected based on yield, rather a single copy clone should be identified. Perhaps highlighted by the fact that multi-copy clones could only be generated with the pZ $\alpha$ GFP vector when starting with a single copy clone (Fig. 4), the underlying cause may be the balance between early growth rate and

Table 2 Time and costs of performing traditional plate PTVA and liquid PTVA for both L12 and L24 with Zeocin and G418 selection [28]

\begin{tabular}{|c|c|c|c|c|c|c|}
\hline & \multicolumn{3}{|l|}{ Zeocin } & \multicolumn{3}{|l|}{ G418 } \\
\hline & Plate PTVA & L12 PTVA & L24 PTVA & Plate PTVA & L12 PTVA & L24 PTVA \\
\hline Time (days) & 45 & $81 / 2$ & 12 & 45 & $81 / 2$ & 12 \\
\hline Cost (USD) & 67.47 & 32.18 & 32.18 & 39.82 & 18.56 & 18.56 \\
\hline
\end{tabular}

Costs were determined based on prices on the Thermo Fisher Scientific website 
selection pressure. It is possible that cells with a higher initial copy number can already survive in the early stages of PTVA because the extra copies of the resistance gene are sufficient to survive the initially low antibiotic concentrations, which decreases the amount of vector amplification in early rounds. However, this may later lead to problems if the vector cannot be amplified fast enough to adapt to higher concentrations of antibiotic when challenged. However, as no investigations have been undertaken to determine the precise mechanism of PTVA, it is difficult to explain why a single copy clone is a more advantageous starting point.

Finally, the choice of selection marker was identified as important. Although multi-copy clones were achieved with both selection markers, using Zeocin resulted in higher maximum copy number strains (Figs. 2, 4). In addition, the combination of the new liquid PTVA method and Zeocin resulted in strains with faster growth rates and overall higher $\mathrm{OD}_{600}$ (Table 1), which could be beneficial when considering volumetric productivity.

\section{Methods}

\section{Media and growth conditions}

Bacterial strains were cultured in Lennox lysogeny broth (LB) medium (1 \% peptone au casein, $0.5 \%$ yeast extract, $0.5 \% \mathrm{NaCl}$ ) and supplemented with either $100 \mu \mathrm{g} \mathrm{mL}$ Zeocin (Life Technologies, Carlsbad, USA) or $50 \mu \mathrm{g} \mathrm{mL}$ Kanamycin (Sigma Aldrich, Dorset, UK). Yeast strains were cultured in a rich YPD medium ( $2 \%$ peptone au casein, $1 \%$ yeast extract, $2 \%$ dextrose). Expression was carried out in buffered minimal glycerol/methanol medium (BMG/BMY; $100 \mathrm{mM}$ potassium phosphate, $\mathrm{pH}$ 6.0, $1.34 \%$ yeast nitrogen base, $4 \times 10^{-5} \%$ d-Biotin, $1 \%$ glycerol or $0.5 \%$ methanol).

\section{Strain construction}

Bacterial recombinant DNA manipulation was carried out in Escherichia coli strain NEB 5- $\alpha$ (New England Biolabs, Hertfordshire, UK). The superfolder-GFP gene of 717 bp was synthesized by GeneArt ${ }^{\mathrm{TM}}$ Gene Synthesis (Thermo Fisher Scientific, Paisley, UK; Additional file 4: Figure S3). Initially the GFP was amplified by PCR using Phusion ${ }^{\circledR}$ High-Fidelity DNA polymerase (New England Biolabs) and primers designed to add the correct restriction sites for subsequent cloning (Thermo Fisher Scientific). The PCR fragments were gel extracted using the Zymoclean ${ }^{\mathrm{TM}}$ Gel DNA Recovery kit (Zymo Research Corporation, Irvine, USA). The vector pPICZ $\alpha$ A (Thermo Fisher Scientific) and the superfolder-GFP were digested with BstBI and Acc65I and ligated to generate the pPICZ-GFP (pZGFP) vector. Alternatively, pPICZ $\alpha$ A and superfolder-GFP were digested with PmlI and Acc65I and ligated to generate the pPICZ $\alpha$-GFP
(pZaGFP) vector. pKAN $\alpha B$ and $\mathrm{pKANB}$ were a kind gift from Geoff and Joan Lin-Cereghino (University of the Pacific) and along with GFP were digested with PmlI and Acc65I and PstI or Acc65I and ligated to form pKANaGFP (pK $\alpha$ GFP) and pKAN-GFP (pKGFP), respectively. Vectors were ligated with T4 DNA Ligase (New England Biolabs) and transformed into NEB 5- $\alpha$ competent cells (New England Biolabs).

For cloning into P. pastoris 5-10 $\mu \mathrm{g}$ of plasmid DNA was linearized with $P m e I$ at a single restriction site within the $A O X 1$ promoter. The vectors were transformed by electroporation according to recommendations in the Pichia Expression manual (Thermo Fisher Scientific) into the $P$. pastoris strain $\Delta k u 70$ (CBS 12694, CBS-KNAW, Fungal Biodiversity Centre, Utretch, The Netherlands) and grown for $3-5$ days at $30{ }^{\circ} \mathrm{C}$ on either $100 \mu \mathrm{g} \mathrm{mL}$ Zeocin (Thermo Fisher Scientific) or $350 \mu \mathrm{g} \mathrm{mL}^{-1} \mathrm{G} 418$ (Biochrom Ltd., Cambridge, UK) depending on the vector used.

\section{Posttransformational vector amplification}

Plate PTVA was performed as indicated by Sunga et al. [15] on plates containing 100, 500, 1000, 1500, 2000, 2500 and $3000 \mu \mathrm{g} \mathrm{mL}^{-1}$ Zeocin or 350, 500, 1000, 1500, 2000, 2500 and $3000 \mu \mathrm{g} \mathrm{mL}^{-1}$ G418. An additional final step of streaking out the final spot onto plates containing either $3000 \mu \mathrm{g} \mathrm{mL}^{-1}$ Zeocin or G418 was included to separate mixed cultures within the spot before analysis. For liquid PTVA cells were grown in $5 \mathrm{~mL}$ of YPD with the starting antibiotic concentration for either 12 or $24 \mathrm{~h}$ at $30{ }^{\circ} \mathrm{C}$ shaking at $250 \mathrm{rpm}$. After the allotted time cells were centrifuged at $4000 \mathrm{rpm}$ at room temperature for $5 \mathrm{~min}$ and the supernatant discarded. The medium was then replaced with the next sequential concentration of antibiotic in YPD and left to grow for either 12 or $24 \mathrm{~h}$ accordingly. This continued until the highest antibiotic concentration was reached. After the final growth period in $3000 \mu \mathrm{g} \mathrm{mL} \mathrm{g}^{-1}$ of the relevant antibiotic, cells were diluted by $10^{5}$ and plated onto a YPD plate containing $3000 \mu \mathrm{g} \mathrm{mL}^{-1}$ antibiotic and left to grow for 3-5 days to obtain single colonies. For both plate PTVA and liquid PTVA a single colony was used for the starting culture.

\section{Copy number analysis}

Genomic DNA was extracted using the DNeasy ${ }^{\circledR}$ Plant Mini Prep Kit (Qiagen, Crawley, UK). Genomic DNA was quantified by Nanodrop ${ }^{\mathrm{TM}}$ (Thermo Fisher Scientific) and normalized to $0.5 \mathrm{ng} \mu \mathrm{L}^{-1}$ using distilled $\mathrm{H}_{2} \mathrm{O}$. Quantitative PCR was run on genomic DNA using SYBR ${ }^{\circledR}$ Green Jumpstart ${ }^{\mathrm{TM}}$ Taq ReadyMix ${ }^{\mathrm{TM}}$ (Sigma Aldrich) in an Eppendorf Mastercycler ${ }^{\circledR}$ ep realplex quantitative cycler (Eppendorf UK Ltd, Histon, UK). Copy number was calculated using a standard curve against known 
concentrations of the plasmid pKAN-GFP. Primers for GFP were ATC CGG ATC ACA TGA AAC GC and AAG CTA ATG GTG CGT TCC TG resulting in a 79 bp amplicon. Cycling conditions were $95^{\circ} \mathrm{C}$ for 5 min followed by 40 cycles of $95^{\circ} \mathrm{C}$ for $30 \mathrm{~s}, 55^{\circ} \mathrm{C}$ for $30 \mathrm{~s}$ and $72{ }^{\circ} \mathrm{C}$ for $30 \mathrm{~s}$ with a melting curve afterwards to ensure a single product was being measured.

\section{GFP expression}

For expression in $P$. pastoris, samples were cultured in 24-deep well plates in $3 \mathrm{~mL}$ of BMG (VWR International Ltd, Lutterworth, UK) and sealed with Breathe-Easy ${ }^{\circledR}$ sealing membrane (Sigma Aldrich). Cells were incubated at $30{ }^{\circ} \mathrm{C}, 216 \mathrm{rpm}$ for $48 \mathrm{~h}$ in BMG to allow growth before being centrifuged at $4000 \mathrm{rpm}$ for $5 \mathrm{~min}$. The supernatant was removed and the medium replaced with $\mathrm{BMY}$ to induce expression. Cultures were left to express at $20{ }^{\circ} \mathrm{C}, 216 \mathrm{rpm}$ for $24 \mathrm{~h}$ before being harvested. GFP expression was measured on the POLARstar ${ }^{\circledR}$ Omega plate reader (BMG Labtech, Ortenberg, Germany) with excitation at $485 / 12 \mathrm{~nm}$ and emission at 520/30 $\mathrm{nm}$. For intracellular expression samples were normalized according to the $\mathrm{OD}_{600}$ of the cultures and corrected for background autofluorescence using a non-transformed control.

\section{Growth curves}

Overnight growth was performed in $50 \mathrm{~mL}$ centrifuge tubes with the lids loosely attached to promote aeration. A single colony was used to inoculate $5 \mathrm{~mL}$ of YPD and cultured for $16 \mathrm{~h}$ at $30{ }^{\circ} \mathrm{C}, 250 \mathrm{rpm}$. A 1/1000 dilution from the overnight culture was used to inoculate a $250 \mathrm{~mL}$ glass baffled flasks containing $25 \mathrm{~mL}$ YPD and cultures were grown at $30{ }^{\circ} \mathrm{C}, 250 \mathrm{rpm}$. $\mathrm{OD}_{600}$ readings were measured in a Jenway Genova spectrophotometer using YPD as a blank. Readings were taken every hour for the first $9 \mathrm{~h}$, and a final reading at $24 \mathrm{~h}$ was also taken. As growth increased cultures were diluted accordingly with YPD prior to the readings.

\section{Statistical analysis}

All analyses were conducted in R [29]. Due to the nonparametric data set the Wilcoxon-Mann-Whitney and Kruskal-Wallis $\mathrm{H}$ tests were applied.

\section{Cost calculation}

Costs for Zeocin/G418 usage was based on list prices from the Thermo Fisher website [28]. Initial costs were determined per $1 \mathrm{~mL}$ of antibiotic. Each step was calculated in terms of the amount of $\mathrm{mL}$ of antibiotic required for a step, $5 \mathrm{~mL}$ for liquid PTVA stages and $15 \mathrm{~mL}$ for plate PTVA. Total volumes were then multiplied by the cost of $1 \mathrm{~mL}$ of antibiotic.

\section{Additional files}

Additional file 1: Figure S1. Schematic representation of the expression vectors pZGFP, pZaGFP, pKGFP and pKaGFP.

Additional file 2: Figure S2. L24 PTVA results in the higher yield of GFP for both pKGFP-1 and pKaGFP-1.

Additional file 3: Table S1. Summary of PTVA strains.

Additional file 4: Figure S3. DNA Sequence of codon optimized superfolded GFP (sGFP).

\section{Abbreviations}

a-MF: a-mating factor; AOX1: alcohol oxidase 1; L12: 12 h liquid PTVA; L24: $24 \mathrm{~h}$ liquid PTVA; OD 600 : optical density at $600 \mathrm{~nm}$; PTVA: posttransformational vector amplification; pKGFP: pKANB + GFP; pKaGFP: pKANBa + GFP; pZGFP: pPICZ + GFP; PZaGFP: pPICZa + GFP.

\section{Authors' contributions}

RA designed and performed the experiments and conceived the manuscript and helped draft the manuscript. KP helped design the experiments and draft the manuscript. All authors read and approved the final manuscript.

\section{Author details}

${ }^{1}$ Department of Life Sciences, Imperial College London, London SW7 2AZ, UK. ${ }^{2}$ Centre for Synthetic Biology and Innovation, Imperial College London, London SW7 2AZ, UK.

\section{Acknowledgements}

The authors would like to thank the Leverhulme Trust for funding (RPG-2013064). The Centre for Biology and Innovation is generously funded by EPSRC. RA would like to thank David Leak for his helpful discussions. We gratefully acknowledge Geoff and Joan Lin-Cereghino from the University of the Pacific for providing the pKanB and pKanBa vectors. We gratefully acknowledge Dr. Scott Robinson for his advice on statistical analysis.

\section{Competing interests}

The authors declare that they have no competing interests.

Received: 8 October 2015 Accepted: 25 January 2016

Published online: 05 February 2016

\section{References}

1. Cregg JM, Barringer KJ, Hessler AY, et al. Pichia pastoris as a host system for transformations. Mol Cell Biol. 1985;5(12):3376-85.

2. Bushell ME, Rowe M, Avignone-Rossa CA, et al. Cyclic fed-batch culture for production of human serum albumin in Pichia pastoris. Biotechnol Bioeng. 2003:82(6):678-83.

3. Macauley-Patrick S, Fazenda ML, McNeil B, et al. Heterologous protein production using the Pichia pastoris expression system. Yeast. 2005;22(4):249-70.

4. Romanos M, Scorer C, Sreekrishna K, et al. The generation of multicopy recombinant strains. In: Higgins DR, Cregg J, editors. Pichia Protocols. New York: Humana Press; 1998. p. 55-72.

5. Aw R, Polizzi KM. Can too many copies spoil the broth? Microb Cell Fact. 2013;12:128.

6. Clare JJ, Rayment FB, Ballantine SP, et al. High-level expression of tetanus toxin fragment $C$ in Pichia pastoris strains containing multiple tandem integrations of the gene. Biotechnology (N Y). 1991;9(5):455-60.

7. Marx H, Mecklenbrauker A, Gasser B, et al. Directed gene copy number amplification in Pichia pastoris by vector integration into the ribosomal DNA locus. FEMS Yeast Res. 2009;9(8):1260-70.

8. Clare JJ, Romanos MA, Rayment FB, et al. Production of mouse epidermal growth factor in yeast: high-level secretion using Pichia pastoris strains containing multiple gene copies. Gene. 1991;105(2):205-12. 
9. Mansur M, Cabello C, Hernandez L, et al. Multiple gene copy number enhances insulin precursor secretion in the yeast Pichia pastoris. Biotechnol Lett. 2005;27(5):339-45.

10. Hohenblum H, Gasser B, Maurer M, et al. Effects of gene dosage, promoters, and substrates on unfolded protein stress of recombinant Pichia pastoris. Biotechnol Bioeng. 2004;85(4):367-75.

11. Love KR, Politano TJ, Panagiotou V, et al. Systematic single-cell analysis of Pichia pastoris reveals secretory capacity limits productivity. PLoS One. 2012;7(6):e37915.

12. Inan M, Aryasomayajula D, Sinha J, et al. Enhancement of protein secretion in Pichia pastoris by overexpression of protein disulfide isomerase. Biotechnol Bioeng. 2006;93(4):771-8.

13. Shen $\mathrm{Q}$, Wu M, Wang HB, et al. The effect of gene copy number and co-expression of chaperone on production of albumin fusion proteins in Pichia pastoris. Appl Microbiol Biotechnol. 2012;96(3):763-72.

14. Lin-Cereghino J, Hashimoto MD, Moy A, et al. Direct selection of Pichia pastoris expression strains using new G418 resistance vectors. Yeast. 2008;25(4):293-9.

15. Sunga AJ, Tolstorukov I, Cregg JM. Posttransformational vector amplification in the yeast Pichia pastoris. FEMS Yeast Res. 2008;8(6):870-6.

16. Zhu T, Guo M, Tang Z, et al. Efficient generation of multi-copy strains for optimizing secretory expression of porcine insulin precursor in yeast Pichia pastoris. J Appl Microbiol. 2009;107:954-63.

17. Norden K, Agemark M, Danielson J, et al. Increasing gene dosage greatly enhances recombinant expression of aquaporins in Pichia pastoris. BMC Biotechnol. 2011;11(1):47.

18. Naatsaari L, Mistlberger B, Ruth C, et al. Deletion of the Pichia pastoris KU70 homologue facilitates platform strain generation for gene expression and synthetic biology. PLoS One. 2012;7(6):e39720.
19. Brodsky JL, Skach WR. Protein folding and quality control in the endoplasmic reticulum: recent lessons from yeast and mammalian cell systems. Curr Opin Cell Biol. 2011;23(4):464-75.

20. Mattanovich D, Gasser B, Hohenblum H, et al. Stress in recombinant protein producing yeasts. J Biotechnol. 2004;113(1-3):121-35.

21. Drocourt D, Calmels T, Reynes JP, et al. Cassettes of the Streptoalloteichus hindustanus ble gene for transformation of lower and higher eukaryotes to phleomycin resistance. Nucleic Acids Res. 1990;18(13):4009.

22. Bar-Nun S, Shneyour Y, Beckmann JS. G-418, an elongation inhibitor of 80 S ribosomes. Biochim Biophys Acta. 1983;741(1):123-7.

23. Rebnegger $C$, Graf $A B$, Valli $M$, et al. Pichia pastoris growth rate regulates protein synthesis and secretion, mating, and stress response. Biotechnol J. 2013;9:511-25.

24. Mondello C, Smirnova A, Giulotto E. Gene amplification, radiation sensitivity and DNA double-strand breaks. Mutat Res. 2010;704(1-3):29-37.

25. Davies J, Jimenez A. A new selective agent for eukaryotic cloning vectors. Am J Trop Med Hyg. 1980;29(5 Suppl):1089-92.

26. Yallop CA, Svendsen I. The effects of G418 on the growth and metabolism of recombinant mammalian cell lines. Cytotechnology. 2001;35(2):101-14.

27. Lanza AM, Kim DS, Alper HS. Evaluating the influence of selection markers on obtaining selected pools and stable cell lines in human cells. Biotechnol J. 2013;8(7):811-21.

28. Thermo Fisher Scientific, Selection Antibiotics. Available from: http:// www.thermofisher.com/uk/en/home/life-science/cell-culture/transfection/selection.html?icid=fr-antibiotics-main. Accessed 01 Sep 2015.

29. R Core Team. R: a language and environment for statistical computing $R$ foundation for statistical computing, Vienna, Austria. Available from: https://www.R-project.org/(2015).

\section{Submit your next manuscript to BioMed Central and we will help you at every step:}

- We accept pre-submission inquiries

- Our selector tool helps you to find the most relevant journal

- We provide round the clock customer support

- Convenient online submission

- Thorough peer review

- Inclusion in PubMed and all major indexing services

- Maximum visibility for your research

Submit your manuscript at www.biomedcentral.com/submit
() Biomed Central 